\title{
Physalis peruviana L. inhibits airway inflammation induced by cigarette smoke and lipopolysaccharide through inhibition of extracellular signal-regulated kinase and induction of heme oxygenase-1
}

\author{
HYUN AH PARK ${ }^{1,2^{*}}$, JAE-WON LEE ${ }^{1 *}$, OK-KYOUNG KWON ${ }^{1}$, GILHYE LEE ${ }^{1,3}$, YOURIM LIM ${ }^{1,4}$, \\ JUNG HEE KIM ${ }^{1}$, JIN-HYUB PAIK ${ }^{5}$, SANGHO CHOI $^{5}$, IMAM PARYANTO ${ }^{6}$, PRASETYAWAN YUNIATO ${ }^{6}$, \\ DOO-YOUNG KIM ${ }^{1}$, HYUNG WON RYU ${ }^{1}$, SEI-RYANG OH ${ }^{1}$, SEUNG JIN LEE ${ }^{2}$ and KYUNG-SEOP AHN ${ }^{1}$ \\ ${ }^{1}$ Natural Medicine Research Center, Korea Research Institute of Bioscience and Biotechnology (KRIBB), Chungbuk 28116; \\ ${ }^{2}$ College of Pharmacy, Chungnam National University, Daejeon 34134; ${ }^{3}$ School of Life Science and Biotechnology, \\ Korea University, Seoul 02841; ${ }^{4}$ College of Pharmacy, Chungbuk National University, Chungbuk 28160; \\ ${ }^{5}$ International Biological Material Research Center, Korea Research Institute of Bioscience and Biotechnology (KRIBB), \\ Daejeon 34141, Republic of Korea; ${ }^{6}$ Center for Pharmaceutical and Medical Technology, \\ The Agency for the Assessment and Application of Technology (BPPT), Tangerang, Banten 15314, Indonesia
}

Received April 4, 2017; Accepted September 8, 2017

DOI: $10.3892 / \mathrm{ijmm} .2017 .3139$

\begin{abstract}
Physalis peruviana L. (PP) is a medicinal herb that has been confirmed to have several biological activities, including anticancer, antioxidant and anti-inflammatory properties. The aim of the present study was to evaluate the protective effect of PP on cigarette smoke (CS)- and lipopolysaccharide (LPS)-induced pulmonary inflammation. Treatment with PP significantly reduced the influx of inflammatory cells in the bronchoalveolar lavage fluid (BALF) and lung of mice with CS- and LPS-induced pulmonary inflammation. PP also decreased the levels of reactive oxygen
\end{abstract}

Correspondence to: Dr Seung Jin Lee, College of Pharmacy, Chungnam National University, 99 Daehak-ro, Yuseong-gu, Daejeon 34134, Republic of Korea

E-mail: s.j.lee@cnu.ac.kr

Dr Kyung-Seop Ahn, Natural Medicine Research Center, Korea Research Institute of Bioscience and Biotechnology, 30 Yeongudanji-ro, Cheongwon-gu, Chungbuk 28116, Republic of Korea

E-mail:ksahn@kribb.re.kr

${ }^{*}$ Contributed equally

Abbreviations: COPD, chronic obstructive pulmonary disease; CS, cigarette smoke; LPS, lipopolysaccharide; BALF, bronchoalveolar lavage fluid; ROS, reactive oxygen species; $\mathrm{TNF}-\alpha$, tumor necrosis factor- $\alpha$; IL-6, interleukin-6; MCP-1, monocyte chemoattractant protein-1; ERK, extracellular signal-regulated kinase; Nrf2, nuclear factor erythroid 2-related factor 2; HO-1, heme oxygenase-1; $\mathrm{PP}$, Physalis peruviana $\mathrm{L}$.

Key words: Physalis peruviana L., chronic obstructive pulmonary disease, airway inflammation, neutrophils, heme oxygenase-1 species (ROS) and pro-inflammatory cytokines, such as tumor necrosis factor- $\alpha$ (TNF- $\alpha$ ) and interleukin-6 (IL-6) in the BALF. PP effectively attenuated the expression of monocyte chemoattractant protein-1 (MCP-1) and the activation of extracellular signal-regulated kinase (ERK) in the lung. In addition, nuclear factor erythroid 2-related factor 2 (Nrf2) activation and heme oxygenase-1 (HO-1) expression were increased by PP treatment. In an in vitro experiment, PP reduced the mRNA expression of TNF- $\alpha$ and MCP-1, and the activation of ERK in CS extract-stimulated A549 epithelial cells. Furthermore, PP increased the activation of Nrf2 and the expression of HO-1 in A549 cells. These findings suggest that PP has a therapeutic potential for the treatment of pulmonary inflammatory diseases, such as chronic obstructive pulmonary disease.

\section{Introduction}

Chronic obstructive pulmonary disease (COPD) is a global public health concern and a major cause of morbidity and mortality worldwide (1). COPD is characterized by oxidative damage and chronic airway inflammation that results in airflow obstruction and emphysema (2). The exposure to cigarette smoke (CS) is the main cause of COPD, as it alters the inflammatory mechanisms by increasing the influx of inflammatory cells, including neutrophils, and the production of inflammatory molecules (3). Recent studies reported that CS contains harmful particles and trace amounts of microbial cell components, including bacterial lipopolysaccharide (LPS), which play an important role in lung diseases and respiratory infections $(4,5)$.

Airway inflammation is one of the major characteristics of COPD and is associated with an increase in inflammatory cell recruitment (6). Neutrophil influx is a major pathophysiological characteristic of COPD, and persistent activation of neutrophils 
leads to lung tissue damage by increasing the production of reactive oxygen species (ROS) and neutrophil elastase (7). Macrophages affect airway inflammation via production of inflammatory cytokines and chemokines in the pathogenesis of COPD (8). High levels of tumor necrosis factor- $\alpha$ (TNF- $\alpha$ ) and interleukin-6 (IL-6) were detected in COPD patients and CS exposure animal models $(9,10)$. Monocyte chemoattractant protein-1 (MCP-1), a member of the C-C chemokine subfamily, is produced by macrophages and airway epithelial cells, and affects the influx of inflammatory cells, including neutrophils, in airway inflammatory response $(11,12)$. The administration of antioxidants effectively ameliorated the airway inflammation with MCP-1 inhibition (13). Downregulation of extracellular signal-regulated kinase (ERK) reduces the production of inflammatory cytokines and chemokines in CS extract (CSE)-stimulated pulmonary epithelial cells and in animal models with CS-induced airway inflammation $(13,14)$. The antioxidant defense protein heme oxygenase-1 (HO-1) exerts a protective effect against CS-induced lung inflammation (15). The levels of inflammatory cell influx and inflammatory molecules are decreased by HO-1 induction in animal models of CS-induced airway inflammation (16).

Cape gooseberry [Physalis peruviana L. (PP)] is a species within the Solanaceae family, which has been widely used in folk medicine (17). PP displays a wide range of biological properties, including neuroprotective, antioxidant and antiinflammatory properties (18-20). However, the protective effect of PP against airway inflammation induced by CS and LPS has not been extensively investigated.

\section{Materials and methods}

Preparation of PP.PP was collected from the Katu Village, Lore Lindu National Park, Central Sulawesi,Indonesia. Plant samples were collected and identified by the Center for Pharmaceutical and Medical Technology (Tangerang, Indonesia) and verified by Herbarium Bogoriense (Bogor, Indonesia). Voucher specimens were recorded as KRIB 0049496 and PMT 1884 have been deposited at the herbarium of the Korea Research Institute of Bioscence and Biotechnolgy and at the Center for Pharmaceutical and Medical Technology and Herbarium Bogoriense. After drying and grinding the leaves of PP, $150 \mathrm{~g}$ of powder was added to $150 \mathrm{ml}$ of methanol and extraction was performed by maceration at room temperature for $18 \mathrm{~h}$. The extract was filtered and concentrated by a rotary evaporator (Laborota 4000; Heidolph, Jakarta, Indonesia) under reduced pressure, thereby obtaining $7.05 \mathrm{~g}$ of PP methanolic extract. In the following experiment, the extract was dissolved in dimethyl sulfoxide (DMSO) to a concentration of $20 \mathrm{mg} / \mathrm{ml}$, and then diluted to various concentrations before use.

Mouse models of airway inflammation induced by CS and LPS. Male 6-week-old C57BL/6N mice were purchased from Koatech Co. (Pyeongtaek, Korea) and used after 1 week of quarantine and acclimatization in a specific pathogen-free system. The mice were randomly divided into 4 groups $(n=7$ per group) as follows: i) The normal control (NC) group; ii) the CS + LPS group; iii) the roflumilast (ROF; $10 \mathrm{mg} / \mathrm{kg}$ ) group (used as a positive control); and iv) the PP group (administered 10 and $20 \mathrm{mg} / \mathrm{kg} \mathrm{PP}$ ). CS exposure was applied as previously described (7). In brief, the mice were whole-body exposed to room air or CS of 8 cigarettes for $1 \mathrm{~h}$ a day for 10 days. CS exposure was generated by $3 \mathrm{R} 4 \mathrm{~F}$ research cigarette, containing $11.0 \mathrm{mg}$ of total particulate matter, $9.4 \mathrm{mg}$ of tar, and $0.76 \mathrm{mg}$ of nicotine/cigarette (Tobacco and Health Research Institute, University of Kentucky, Lexington, KY, USA). ROF and PP were dissolved with $1 \%$ DMSO $+1 \%$ Tween-20 in phosphate-buffered saline (PBS), and were administered orally on days $0-9$. The mice were administered LPS (5 $\mu \mathrm{g}$ dissolved in $30 \mu \mathrm{l}$ distilled water) intranasally $1 \mathrm{~h}$ after the final ROF and PP treatment. All the experimental procedures were performed in accordance with the procedures approved by the Institutional Animal Care and Use Committee of the Korea Research Institute of Bioscience and Biotechnology, and in compliance with the National Institutes of Health Guidelines for the Care and Use of Laboratory Animals and Korean National Laws for Animal Welfare.

Collection of bronchoalveolar lavage fluid (BALF) and determination of inflammatory cell counts. The collection of the BALF and determination of inflammatory cell counts were performed as previously described (13). In brief, ice-cold PBS (700 $\mu \mathrm{l})$ was infused into the lungs via tracheal cannulation and extraction was performed twice (total volume, 1,400 $\mu \mathrm{l}$ ). In order to count the number of different cells, $100 \mu \mathrm{l}$ BALF was centrifuged onto glass slides for $5 \mathrm{~min}$ at $264 \mathrm{x} \mathrm{g}$, and the slides were dried and stained using Diff-Quik ${ }^{\circledR}$ staining reagent according to the manufacturer's protocol (IMEB Inc., Deerfield, IL, USA).

Measurement of ROS and pro-inflammatory cytokines in the $B A L F$. The intracellular levels of ROS were determined using 2',7'-dichlorofluorescein diacetate (DCF-DA; Sigma-Aldrich, St. Louis, MO, USA) as previously described (21). Briefly, BALF cells were washed with PBS and incubated with $20 \mu \mathrm{M}$ DCF-DA for $10 \mathrm{~min}$ at $37^{\circ} \mathrm{C}$. Subsequently, the intracellular ROS levels were detected under fluorescence at $488 \mathrm{~nm}$ excitation and $525 \mathrm{~nm}$ emission on a fluorescence plate reader (Perkin-Elmer, Waltham, MA, USA). The levels of pro-inflammatory cytokines (TNF- $\alpha$, IL-6 and MCP-1) were determined using enzyme-linked immunosorbent assay (ELISA) kits according to the manufacturer's protocol (R\&D Systems, Minneapolis, MN, USA). The absorbance was measured at $450 \mathrm{~nm}$ using a microplate reader (Molecular Devices, Sunnyvale, CA, USA).

Western blot analysis. The lung tissues were collected at 6 or $24 \mathrm{~h}$ after the last administration of PP. The levels of ERK and nuclear factor erythroid 2-related factor 2 (Nrf2) activation were evaluated using lung tissues that were obtained $6 \mathrm{~h}$ after the last administration of PP. The expression of MCP-1, inducible nitric oxide synthase (iNOS), cyclooxygenase-2 (COX-2) and HO-1 were evaluated using lung tissues that were collected $24 \mathrm{~h}$ after the last administration of PP. The lung tissues were homogenized $(1 / 10, \mathrm{w} / \mathrm{v})$ in tissue lysis/extraction reagent, containing a protease inhibitor cocktail (Sigma-Aldrich). Equal amounts of the total cellular protein were resolved by 8-12\% SDS-polyacrylamide gels and transferred to Hybond PVDF membrane (Amersham Biosciences, Piscataway, NJ, USA). The membranes were incubated in blocking solution [5\% skimmed milk in Tris-buffered saline $+0.1 \%$ Tween-20 
(TBST)] for $1 \mathrm{~h}$ and incubated overnight at $4^{\circ} \mathrm{C}$ with the appropriate primary antibody. A rabbit polyclonal MCP-1 antibody (sc-28879, 1:1,000; Santa Cruz Biotechnology, Inc., Santa Cruz, CA, USA), a rabbit polyclonal p-ERK antibody (cat. no. 9101, 1:1,000; Cell Signaling Technology Inc., Danvers, MA, USA), a rabbit polyclonal iNOS antibody (SC-651, 1:1,000; Santa Cruz Biotechnology, Inc.), a goat polyclonal COX-2 antibody (SC-1747, 1:1,000), a rabbit polyclonal ERK antibody (sc-154, 1:1,000), a rabbit polyclonal Nrf2 antibody (sc-722, 1:1,000), a rabbit polyclonal p-Nrf2 antibody (ab76026, 1:1,000; Abcam, Cambridge, UK), a rabbit polyclonal HO-1 antibody (cat. no. 5061, 1:1,000), and a rabbit polyclonal anti- $\beta$-actin antibody (cat. no. 4967, 1:2,500) (both from Cell Signaling Technology Inc.) were diluted in 5\% skimmed milk. The membranes were washed in TBST and incubated with the Peroxidase-AffiniPure goat anti-rabbit $\operatorname{IgG}(\mathrm{H}+\mathrm{L})$ (111-035-003 1:2,000; Jackson ImmunoResearch Laboratories, Inc., West Grove, PA, USA) and the Peroxidase-AffiniPure goat anti-mouse IgG (H+L) (115-035-003, 1:2,000; Jackson ImmunoResearch Laboratories, Inc.) for $2 \mathrm{~h}$ at room temperature. The membranes were washed with TBST and developed using an enhanced chemiluminescence kit (Thermo Fisher Scientific, Inc., Rockford, IL, USA). All bands were visualized using a LAS-4000 luminescent image analyzer (Fujifilm, Tokyo, Japan) and quantified by densitometry (Fuji Multi Gauge software, version 3.0).

CSE-stimulated inflammatory molecules in human airway epithelial cells. A549 human airway epithelial cells were obtained from the American Type Culture Collection (Manassas, VA, USA) and were grown in RPMI-1640 medium (Thermo Fisher Scientific, Inc., Carlsbad, CA, USA) supplemented with $10 \%$ FBS in the presence of penicillin $(100 \mathrm{U} / \mathrm{ml})$ and streptomycin $(100 \mu \mathrm{g} / \mathrm{ml})$, and were incubated in a $5 \% \mathrm{CO}_{2}$ incubator. CSE was purchased from Kentucky Reference 3R4F research blend cigarettes (University of Kentucky) and administered as previously described (22). The A549 human lung epithelial cells were incubated with the indicated concentration of PP prior to the addition of CSE $(10 \mu \mathrm{g} / \mathrm{ml})$.

Reverse transcription-polymerase chain reaction (RT-PCR). The A549 cells were treated with PP in the absence or presence of CSE $(10 \mu \mathrm{g} / \mathrm{ml})$ for $6 \mathrm{~h}$. Total RNA was isolated using TRIzol $^{\text {TM }}$ reagent and reverse-transcribed into cDNA, according to the manufacturer's protocol (Thermo Fisher Scientific, Inc.). The PCR conditions for MCP- 1 were as follows: $94^{\circ} \mathrm{C}$ for $10 \mathrm{~min}$ (1 cycle), $94^{\circ} \mathrm{C}$ for $30 \mathrm{sec}, 58^{\circ} \mathrm{C}$ for $30 \mathrm{sec}$, and $72^{\circ} \mathrm{C}$ for $1 \mathrm{~min}$ ( 25 cycles); for TNF- $\alpha, 94^{\circ} \mathrm{C}$ for $10 \mathrm{~min}(1 \mathrm{cycle}), 94^{\circ} \mathrm{C}$ for $30 \mathrm{sec}, 57^{\circ} \mathrm{C}$ for $30 \mathrm{sec}$, and $72^{\circ} \mathrm{C}$ for $1 \mathrm{~min}(25$ cycles); for $\beta$-actin, $94^{\circ} \mathrm{C}$ for $10 \mathrm{~min}(1 \mathrm{cycle}), 94^{\circ} \mathrm{C}$ for $30 \mathrm{sec}, 59^{\circ} \mathrm{C}$ for $30 \mathrm{sec}$, and $72^{\circ} \mathrm{C}$ for $1 \mathrm{~min}$ ( $\left.25 \mathrm{cycles}\right)$; and then a final extension phase at $72^{\circ} \mathrm{C}$ for $10 \mathrm{~min}$. The specific forward and reverse primers for TNF- $\alpha$ and MCP-1 were as follows: TNF- $\alpha$ forward, 5'-TCAACCTCCTCTCTGCCATC-3' and reverse, 5'-CCTAAG CCCCCAATTCTCTT-3'; MCP-1 forward, 5'-TCTGTGCCTG CTGCTCATAG-3' and reverse, 5'-CAGATCTCCTTGGCCAC AAT-3'; and $\beta$-actin forward, 5'-CATGTACGTTGCTATCCA GGC-3' and reverse, 5'-CTCCTTAATGTCACGCACGAT-3'. The housekeeping gene $\beta$-actin was used for normalization of RT-PCR. The PCR products were separated on agarose gel.
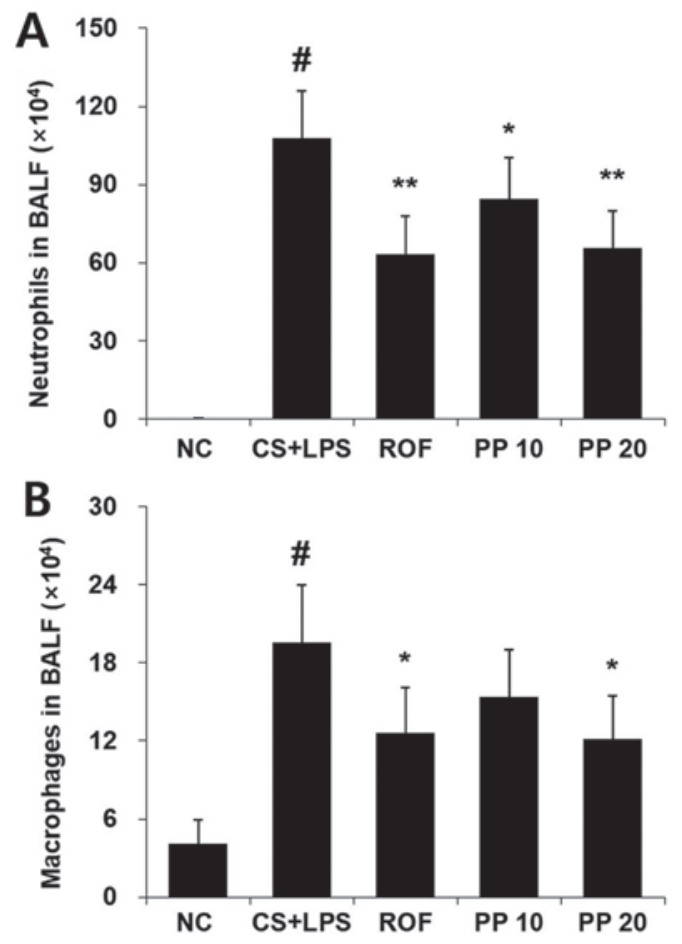

Figure 1. Physalis peruviana L. (PP) suppresses the recruitment of inflammatory cells, such as (A) neutrophils and (B) macrophages in the bronchoalveolar lavage fluid (BALF) of mice with cigarette smoke (CS)- and lipopolysaccharide (LPS)-induced inflammation. BALF differential cell count was evaluated with the Diff-Quik ${ }^{\circledR}$ staining reagent. NC, normal control mice; CS + LPS, mice exposed to CS and LPS; ROF, mice administered roflumilast $(10 \mathrm{mg} / \mathrm{kg})+\mathrm{CS}+\mathrm{LPS}$; PP 10 and 20, mice administered 10 or $20 \mathrm{mg} / \mathrm{kg} \mathrm{PP}+\mathrm{CS}+$ LPS. Data are expressed as the means \pm standard deviation. ${ }^{~} \mathrm{P}<0.01$ indicating a statistically significant difference from the NC group; ${ }^{*} \mathrm{P}<0.05$ and ${ }^{* *} \mathrm{P}<0.01$ indicating statistically significant differences from the CS + LPS group.

Histological analysis. To evaluate the protective effect of PP, lung tissues were obtained at $24 \mathrm{~h}$ after PP administration, washed with PBS and fixed in $10 \%(\mathrm{v} / \mathrm{v})$ neutral buffered formalin solution. The lung tissues were then embedded in paraffin, sectioned at $4 \mu \mathrm{m}$ using a rotary microtome, and stained with hematoxylin and eosin (H\&E; Sigma-Aldrich) to estimate inflammatory response.

Statistical analysis. The data are expressed as the means \pm standard deviation obtained from at least three independent experiments. Statistical significance was determined using two-tailed Student's t-test. A value of $\mathrm{P}<0.05$ was considered to indicate statistically significant differences.

\section{Results}

Treatment with PP attenuates the recruitment of neutrophils in the BALF of CS- and LPS-induced airway inflammation animal models. The increased number of neutrophils and macrophages in the BALF is a cornerstone characteristic of CS- and LPS-induced airway inflammation. Thus, the effect of PP on the infiltration of these cells was evaluated using Diff-Quik ${ }^{\circledR}$ staining. As shown in Fig. 1, increased numbers of neutrophils and macrophages were detected in the CS + LPS group, whereas decreased numbers of these cells were detected in the PP group, and this effect was 
A

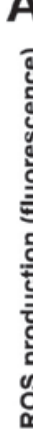

5,000

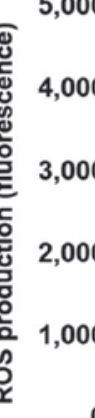

B

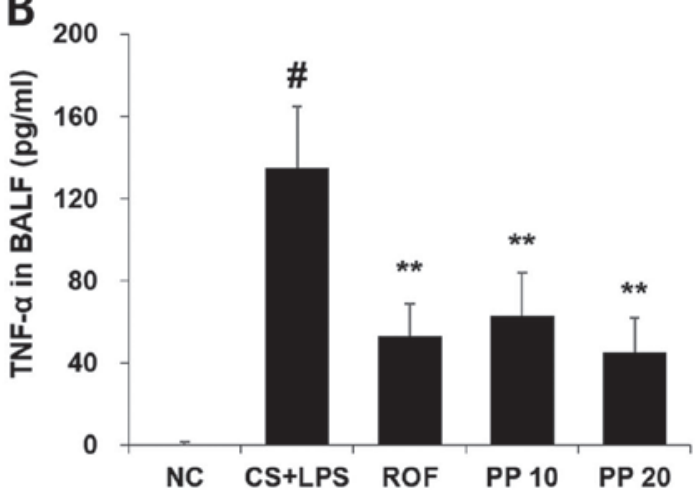

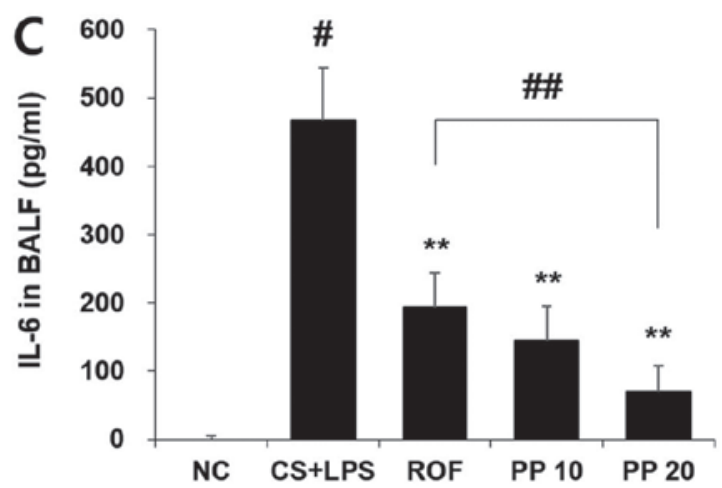

Figure 2. Physalis peruviana L. (PP) decreases the levels of reactive oxygen species (ROS) and pro-inflammatory cytokines, such as tumor necrosis factor- $\alpha$ (TNF- $\alpha$ ) and interleukin (IL)-6 in the bronchoalveolar lavage fluid (BALF) of mice with cigarette smoke (CS)- and lipopolysaccharide (LPS)-induced inflammation. (A) The production of ROS was determined using 2',7'-dichlorofluorescein diacetate. (B and C) The levels of TNF- $\alpha$ and IL-6 were measured by enzyme-linked immunosorbent assay (ELISA). The absorbance was measured at $450 \mathrm{~nm}$ using a microplate reader. NC, normal control mice; CS + LPS, mice exposed to CS and administered LPS; ROF, mice exposed CS and administered roflumilast (10 mg/kg) + LPS; PP 10 and 20 , mice exposed to CS and administered 10 or $20 \mathrm{mg} / \mathrm{kg}$ PP + LPS. Data are expressed as the means \pm standard deviation; ${ }^{\text {P }}<0.01$ indicates a statistically significant difference from the NC group; ${ }^{*} \mathrm{P}<0.05$ and ${ }^{* *} \mathrm{P}<0.01$ indicate statistically significant differences from the CS + LPS group; ${ }^{\#} \mathrm{P}<0.01$ indicates statistically significant differences from the ROF group.

concentration-dependent (Fig. 1). ROF was used as positive control. The effect of $20 \mathrm{mg} / \mathrm{kg}$ PP was similar to that of treatment with $10 \mathrm{mg} / \mathrm{kg}$ ROF.

Treatment with PP reduces the levels of ROS and pro-inflammatory cytokines in the BALF. Intranasal administration of LPS markedly increased the levels of ROS and inflammatory cytokines, including TNF- $\alpha$ and IL-6. However, treatment with PP significantly reduced these levels in a concentrationdependent manner (Fig. 2). In particular, $20 \mathrm{mg} / \mathrm{kg} \mathrm{PP}$ attenuated the production of IL- 6 more effectively compared with the ROF or CS + LPS groups (Fig. 2C).

Treatment with PP inhibits the infiltration of inflammatory cells and the expression of MCP-1 in the lungs. It was next examined whether PP affects the infiltration of inflammatory cells and the production of MCP-1 in the lungs of animal models with CS- and LPS-induced airway inflammation. As shown in Fig. 3A, increased influx of inflammatory cells was observed around peribronchial lesions in the CS + LPS group, whereas a significant reduction of the influx of these cells was detected in the PP group. Treatment with PP also significantly attenuated the expression of MCP-1 compared with the ROF or CS + LPS groups (Fig. 3B). Similar to the results obtained by $\mathrm{PP}$ in the lung, a decreased level of MCP-1 in the BALF was confirmed following PP administration (Fig. 3C).
Treatment with PP decreases the expression of iNOS and $C O X-2$, and reduces the activation of ERK in the lung. LPS administration markedly increased the expression of iNOS and COX-2 compared with the NC group (Fig. 4). However, treatment with PP significantly decreased the levels of iNOS and COX-2 compared with the CS + LPS group in a concentration-dependent manner (Fig. 4A). Similar to the results for iNOS and COX-2, ERK phosphorylation was also effectively decreased with PP administration. In particular, the effect of $20 \mathrm{mg} / \mathrm{kg}$ PP was similar to that of $10 \mathrm{mg} / \mathrm{kg} \mathrm{ROF}$ (Fig. 4B).

Treatment with PP increases the expression of $\mathrm{HO}-1$ in the lungs. HO-1 induction exerts a protective effect in airway inflammatory diseases, including COPD. Thus, western blot analysis was used to investigate whether PP promotes the expression of HO-1. There was an increase in HO-1 expression in the PP group compared with the $\mathrm{NC}$ or $\mathrm{ROF}$ groups (Fig. 5B). Activation of Nrf2, which is a major transcription factor of $\mathrm{HO}-1$, was also observed following PP administration (Fig. 5A).

Pretreatment with PP reduces the $m R N A$ expression of inflammatory molecules and the activation of ERK in A549 airway epithelial cells. CSE treatment significantly increased the mRNA expression of TNF- $\alpha$ and MCP-1 in A549 cells. However, these effects were effectively attenuated by PP 

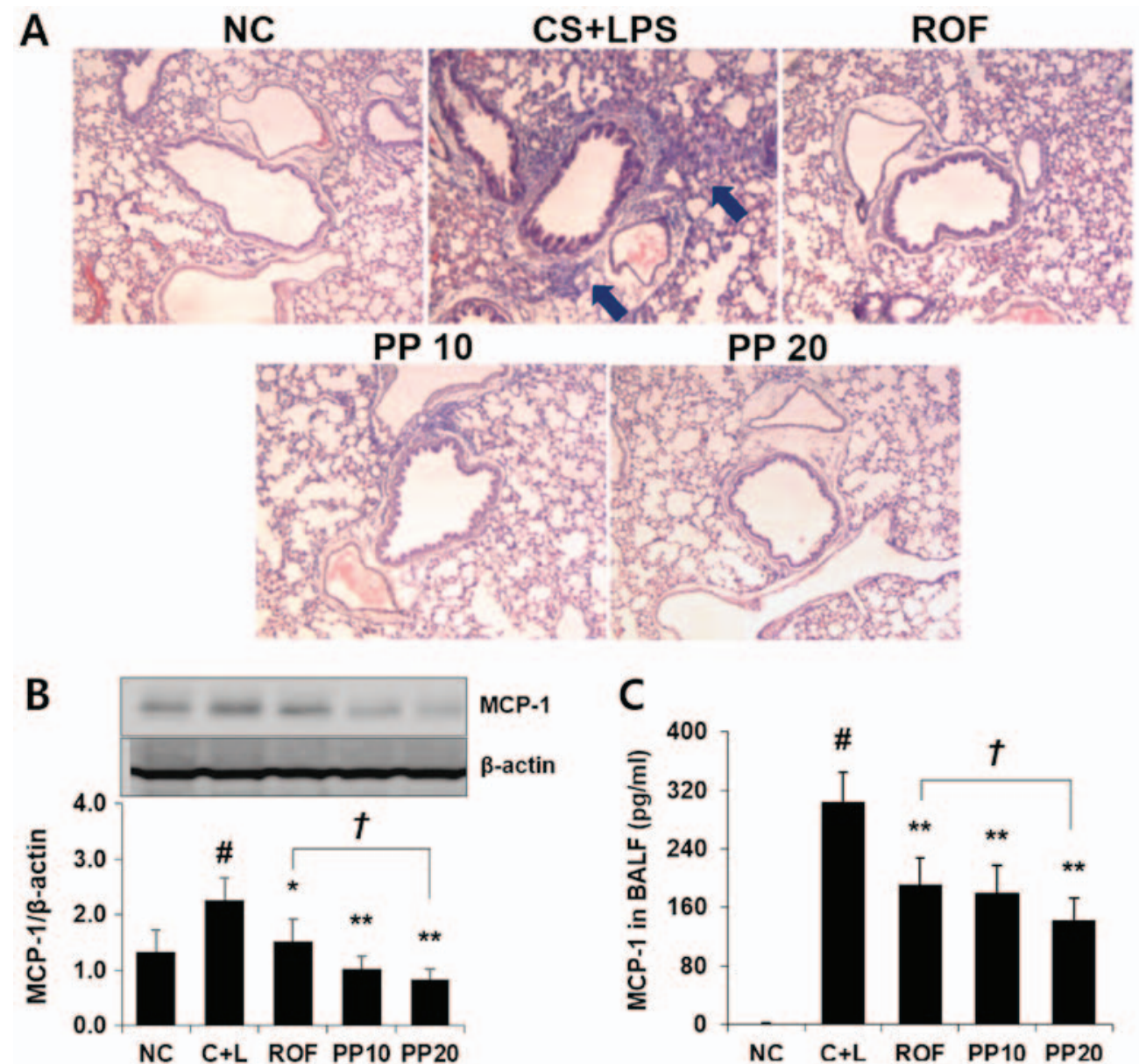

Figure 3. Physalis peruviana L. (PP) inhibits the influx of inflammatory cells and the expression of monocyte chemoattractant protein-1 (MCP-1) in the lungs of mice with cigarette smoke (CS)- and lipopolysaccharide (LPS)-induced inflammation. (A) A peribronchial lesion was stained with hematoxylin and eosin to evaluate the airway inflammation (magnification, x200). (B and C) The levels of MCP-1 were detected using western blot analysis and enzyme-linked immunosorbent assay (ELISA). NC, normal control mice; CS + LPS, mice exposed to CS and administered LPS; ROF, mice exposed to CS and administered roflumilast $(10 \mathrm{mg} / \mathrm{kg})+\mathrm{LPS}$; PP 10 and 20, mice exposed to CS and administered 10 or $20 \mathrm{mg} / \mathrm{kg} \mathrm{PP}+\mathrm{LPS}$. Data are expressed as the means \pm standard deviation; ${ }^{*} \mathrm{P}<0.05$ indicates a statistically significantly difference from the $\mathrm{NC}$ group; ${ }^{*} \mathrm{P}<0.05$ and ${ }^{* *} \mathrm{P}<0.01$ indicate statistically significant differences from the CS + LPS group; ${ }^{\dagger} \mathrm{P}<0.05$ indicates statistically significant differences from the ROF group.
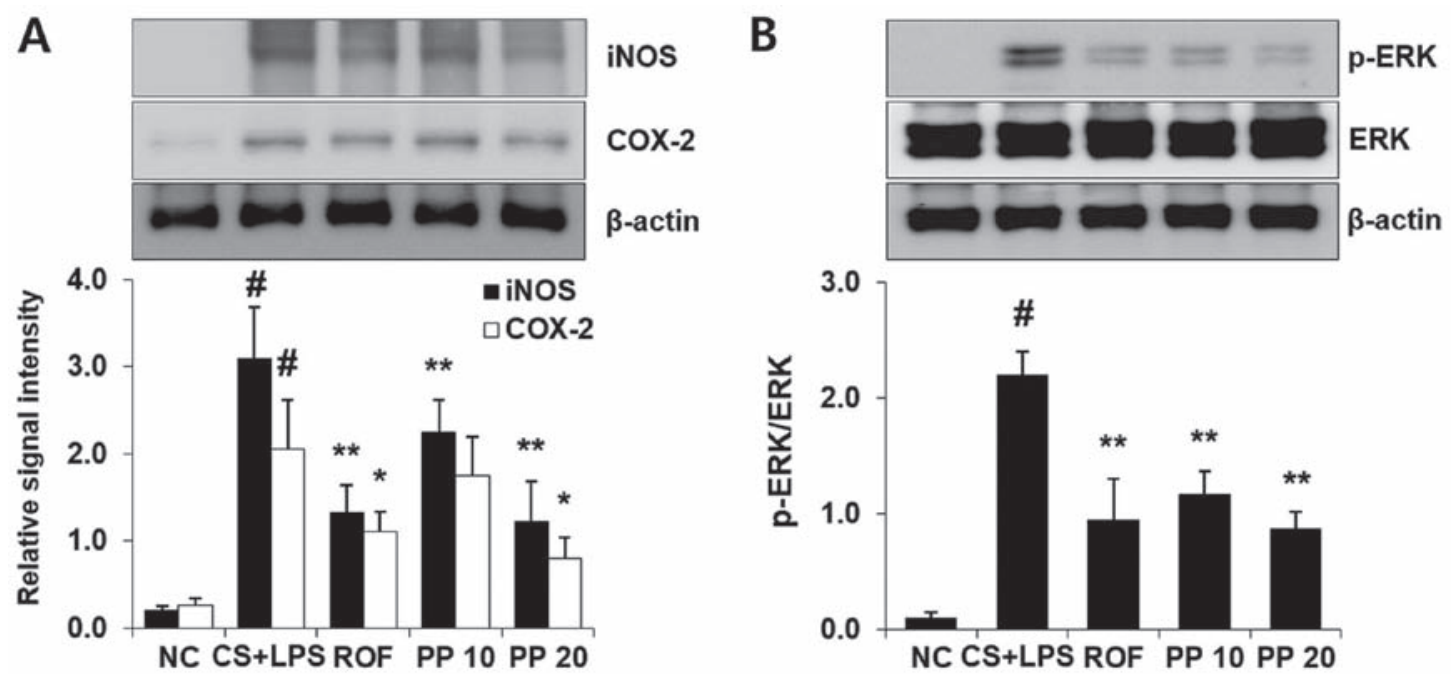

Figure 4. (A and B) Physalis peruviana L. (PP) suppresses the expression of inducible nitric oxide synthase (iNOS) and cyclooxygenase-2 (COX-2), and the activation of extracellular signal-regulated kinase (ERK) in the lungs of mice with cigarette smoke (CS)- and lipopolysaccharide (LPS)-induced inflammation. The levels of iNOS, COX-2 and phosphorylated ERK expression were measured by western blot analysis. Quantitative analysis was performed by a densitometric method. $\beta$-actin was used as an internal control. NC, normal control mice; CS + LPS, mice exposed to CS and administered LPS; ROF, mice exposed to CS and administered roflumilast $(10 \mathrm{mg} / \mathrm{kg})+\mathrm{LPS}$; PP 10 and 20, mice exposed to CS and administered $10 \mathrm{or} 20 \mathrm{mg} / \mathrm{kg}$ PP + LPS. Data are expressed as the means \pm standard deviation; ${ }^{~} \mathrm{P}<0.01$ indicates a statistically significant difference from the $\mathrm{NC}$ group; ${ }^{*} \mathrm{P}<0.05$ and ${ }^{* *} \mathrm{P}<0.01$ indicate statistically significant differences from the CS + LPS group. 

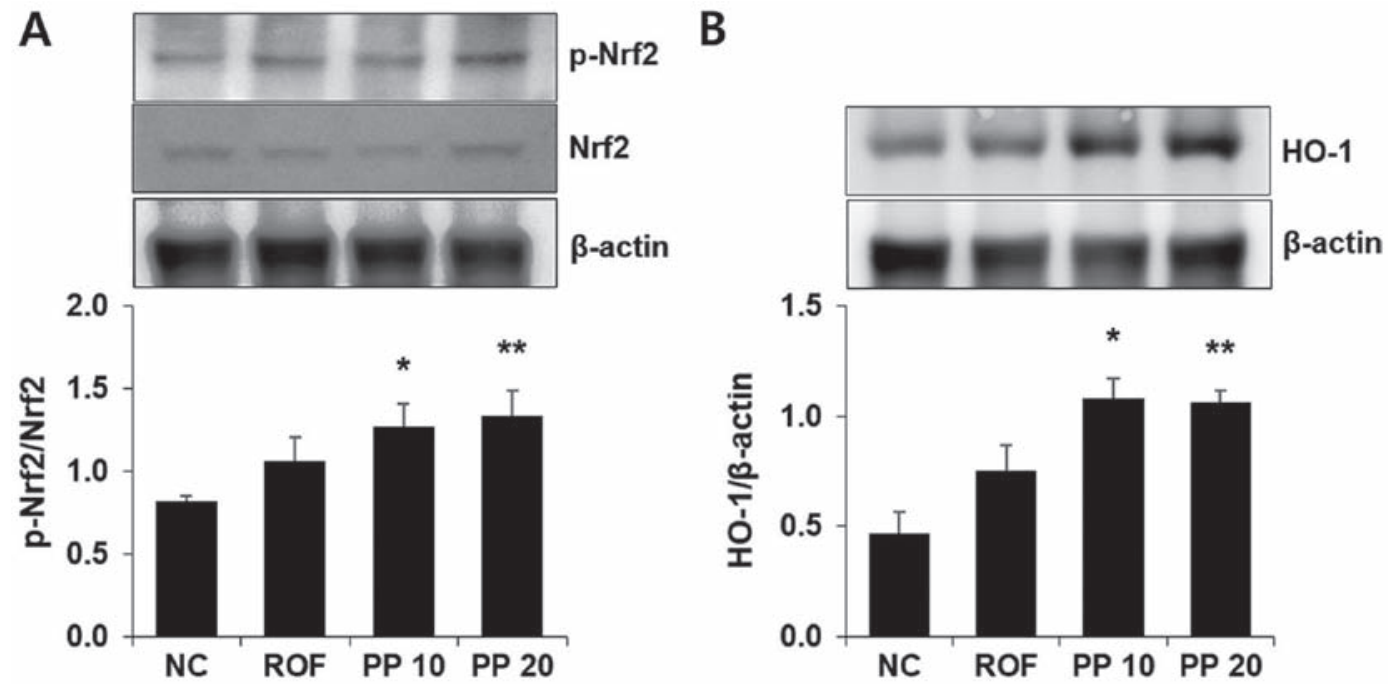

Figure 5. Physalis peruviana L. (PP) upregulates the expression of heme oxygenase-1 (HO-1) in the lungs of mice with cigarette smoke (CS) and lipopolysaccharide (LPS)-induced inflammation. (A) Nuclear factor erythroid 2-related factor 2 (Nrf2) activation and (B) HO-1 expression in the lungs were measured by western blot analysis. Quantitative analysis was performed by a densitometric method. $\beta$-actin was used as an internal control. $\mathrm{NC}$, normal control mice; CS + LPS, mice exposed to CS and administered LPS; ROF, mice exposed to CS and administered roflumilast $(10 \mathrm{mg} / \mathrm{kg})+\mathrm{LPS} ;$ PP 10 and 20 , mice exposed to CS and administered 10 or $20 \mathrm{mg} / \mathrm{kg}$ PP + LPS. Data are expressed as the means \pm standard deviation; ${ }^{*} \mathrm{P}<0.05$ and ${ }^{* *} \mathrm{P}<0.01$ indicated statistically significant differences from the NC group.
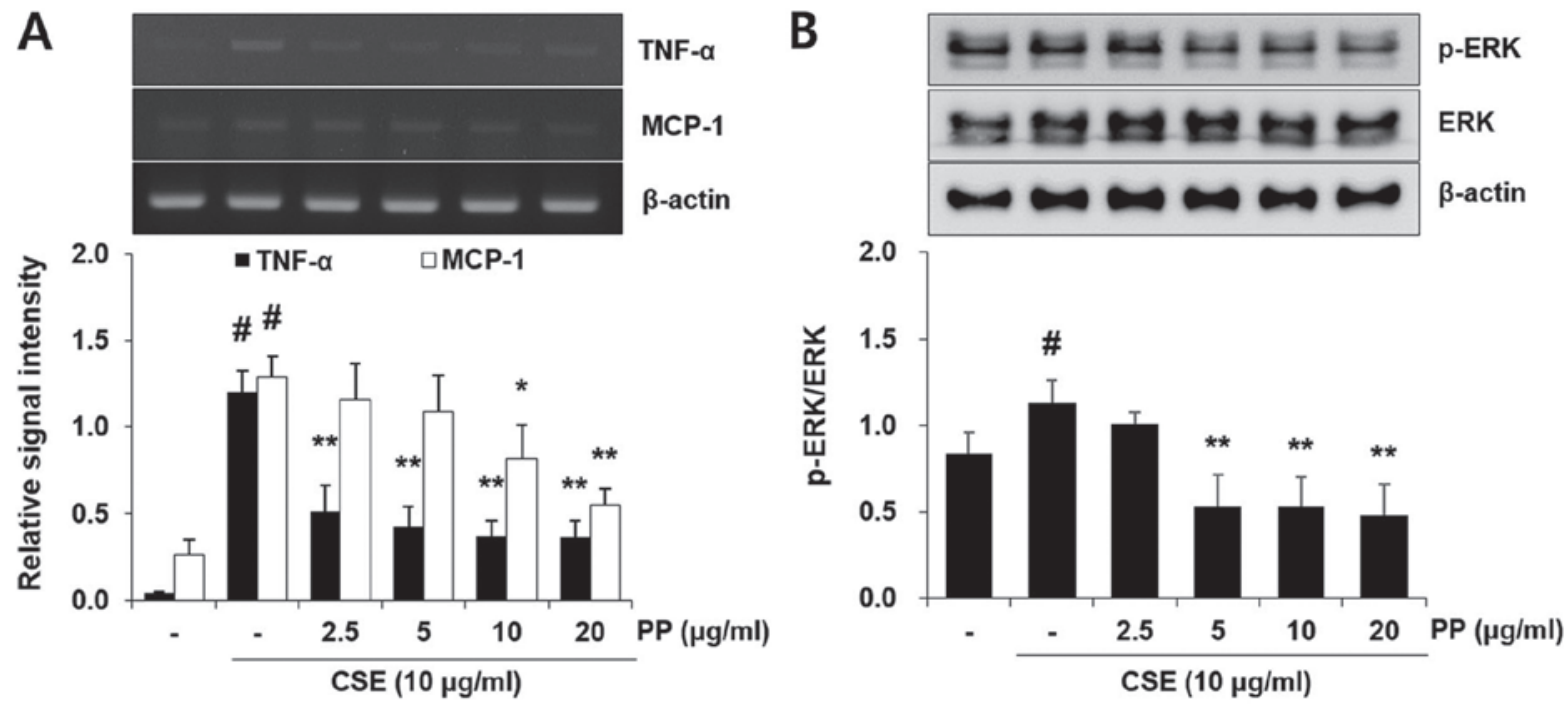

Figure 6. Physalis peruviana L. (PP) downregulates the levels of inflammatory molecules and extracellular signal-regulated kinase (ERK) activation in cigarette smoke (CS) extract (CSE)-stimulated A549 human airway epithelial cells. (A) The levels of tumor necrosis factor- $\alpha$ (TNF- $\alpha$ ) and monocyte chemoattractant protein-1 (MCP-1) mRNA were determined using reverse transcription-polymerase chain reaction. (B) The phosphorylation of ERK was determined with western blot analysis. Data are expressed as the means \pm standard deviation; ${ }^{*} \mathrm{P}<0.05$ indicates a statistically significantly difference from the normal control group; ${ }^{*} \mathrm{P}<0.05$ and ${ }^{* *} \mathrm{P}<0.01$ indicate statistically significant differences from the CS + LPS group. LPS, lipopolysaccharide.

pretreatment (Fig. 6A). It was also confirmed that pretreatment with PP significantly decreased the activation of ERK in CSE-stimulated A549 cells (Fig. 6B). In addition, PP increased Nrf2 activation and HO-1 expression in A549 cells (Fig. 7).

\section{Discussion}

COPD is respiratory condition characterized by expiratory airway obstruction, the major cause of which is chronic airway inflammation (23). Given the importance of airway inflammation in COPD, the anti-inflammatory activity of PP was investigated using CS- and LPS-induced airway inflammation animal models. As increased influx of neutrophils is a cornerstone characteristic of airway inflammation in COPD, it was investigated whether treatment with PP attenuates this influx in the airway inflammatory response. As shown in Fig. 1A, there was a distinct reduction in the neutrophil influx in the BALF of the PP group compared with the CS + LPS group. In particular, the effect of $20 \mathrm{mg} / \mathrm{kg}$ PP was similar to that of $10 \mathrm{mg} / \mathrm{kg}$ ROF. Oxidative stress is implicated in airway inflammation and recognized as a one of the major predicting factors in the pathogenesis of COPD (24). Increased amounts of ROS are generated by neutrophils against CS and 


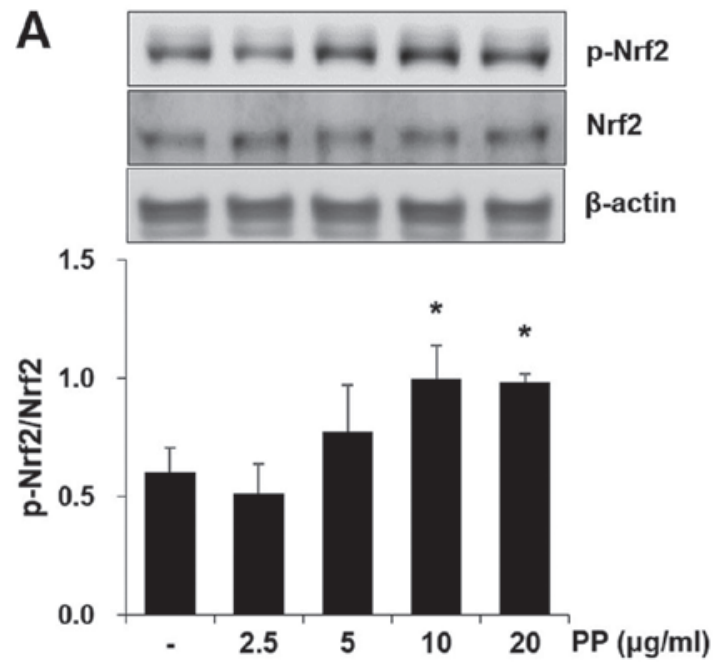

B
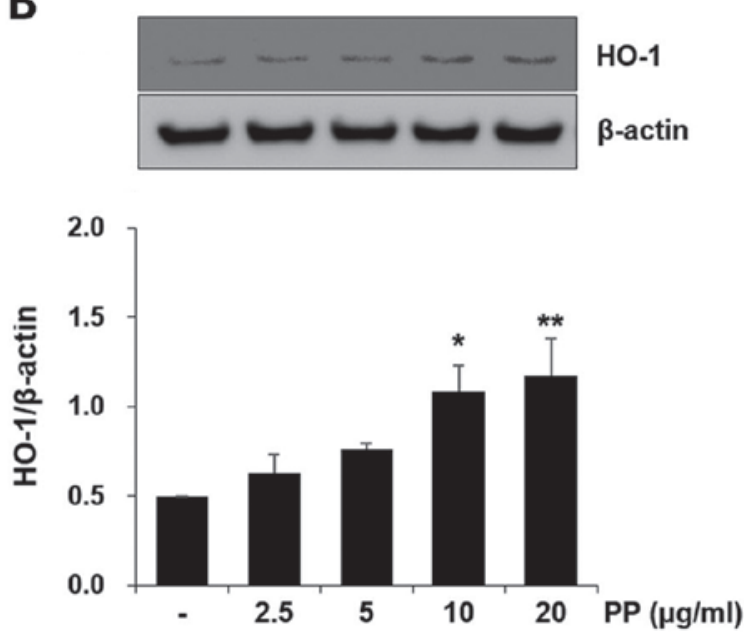

Fiugre 7. Physalis peruviana L. (PP) upregulates the expression of heme oxygenase-1 (HO-1) in A549 human airway epithelial cells. (A) Nuclear factor erythroid 2-related factor 2 (Nrf2) activation and (B) HO-1 expression in A549 cells were determined by western blot analysis. Quantitative analysis was performed by a densitometric method. $\beta$-actin was used as an internal control. Data are expressed as the means \pm standard deviation; ${ }^{*} \mathrm{P}<0.05$ and ${ }^{* *} \mathrm{P}<0.01$ indicate statistically significant differences from the normal control group.

contribute to oxidative stress (25). Therefore, it was investigated whether PP inhibits the increase in the level of ROS. As shown in Fig. 2A, ROS production was upregulated in the BALF of the CS + LPS group compared with the NC group $(\mathrm{P}<0.01)$, whereas treatment with PP significantly decreased ROS production in a concentration-dependent manner, indicating that PP exerts an antioxidant effect in the airway inflammatory response (Fig. 2A). Similar to the results for ROS, PP effectively attenuated the levels of TNF- $\alpha$ and IL-6 in the BALF (Fig. 2B and C). In particular, $20 \mathrm{mg} / \mathrm{kg}$ PP inhibited the production of IL-6 more effectively compared with $10 \mathrm{mg} / \mathrm{kg}$ ROF (Fig. 2C). The inhibition rates of IL-6 production were $58.6 \%$ (ROF), $69.0 \%(\mathrm{PP} 10 \mathrm{mg} / \mathrm{kg}$ ) and $84.9 \%$ (PP $20 \mathrm{mg} / \mathrm{kg}$ ). Increased recruitment of inflammatory cells into the lung is the major pathophysiological mechanism underlying COPD (26). Based on this result, H\&E staining was used to determine whether PP exerts an inhibitory effect on the recruitment of inflammatory cells. As shown in Fig. 3A, exposure to CS and LPS markedly upregulated the recruitment of inflammatory cells, whereas treatment with PP markedly reduced this recruitment (Fig. 3A). These results indicate that PP plays an antioxidant and anti-inflammatory role in airway inflammation induced by CS and LPS.

$\mathrm{Ng}$ et al demonstrated that the inhibition of MCP-1 may be valuable in the inhibition of CS-induced airway inflammation (27). Our recent study also confirmed that antioxidant treatment attenuates airway inflammation induced by CS through the inhibition of MCP-1 (13). As airway inflammatory response is accompanied by increased production of MCP-1, it was investigated whether PP acts as a MCP-1 inhibitor. As shown in Fig. 3B, treatment with PP significantly decreased the expression of MCP-1 compared with the CS + LPS group, in a concentration-dependent manner $(\mathrm{P}<0.01)$ (Fig. 3B). Similar to those results, the increased level of MCP-1 was effectively reduced with PP administration (Fig. 3C). In particular, $20 \mathrm{mg} / \mathrm{kg}$ PP decreased these levels more effectively compared with the ROF or CS + LPS groups (Fig. 3B and C). These results indicate that $\mathrm{PP}$ affects the influx of inflammatory cells as well as the expression of MCP-1, suggesting that PP may be a useful inhibitor of MCP-1.

High levels of iNOS are associated with a variety of pathological conditions, including COPD (28). Gupta et al reported that CS exposure leads to iNOS expression, which results in production of toxic NO metabolites, leading to severe lung damage (29). The levels of COX-2 are significantly increased in small airway epithelium of patients with COPD, and the increase in the levels of this molecule contribute to airway remodeling and inflammation, which are major characteristics of COPD (30). Therefore, inhibition of iNOS and COX-2 may be important for reducing airway inflammation. In the present study, there was significant increase in iNOS and COX-2 expression in the CS + LPS group compared with the NC group $(\mathrm{P}<0.01)$. However, treatment with PP significantly decreased these levels in a concentration-dependent manner (Fig. 4A and B). The inhibitory effect of $20 \mathrm{mg} / \mathrm{kg}$ PP on these molecules was similar to that of $10 \mathrm{mg} / \mathrm{kg}$ ROF.

The ERK signaling cascade is involved in the airway inflammatory response. Li et al reported that CSE causes an inflammatory response through the activation of ERK in human bronchial epithelial cells (31). In our recent study, it was also confirmed that the increased levels of inflammatory cytokines, such as TNF- $\alpha$ and IL-6, were attenuated with downregulation of ERK activation in CSE-stimulated human airway epithelial cells (22). In an in vivo study in airway inflammation animal models, treatment with antioxidants effectively inhibited airway inflammation via inhibition of ERK activation $(13,32)$. Therefore, controlling the activity of the ERK pathway may be a valuable therapeutic approach to airway inflammatory diseases, including COPD. Thus, the effect of PP on ERK activation was evaluated. As shown in Fig. 4, the phosphorylation of ERK was markedly increased in the CS + LPS group, whereas decreased phosphorylation was detected in the PP group. This result indicates that PP may be a useful inhibitor of ERK activation in airway inflammation.

Antioxidant enzymes, such as $\mathrm{HO}-1, \mathrm{NAD}(\mathrm{P}) \mathrm{H}$ quinone oxidoreductase 1 and superoxide dismutase exert protective 
effects against endotoxin-induced inflammation $(33,34)$. Among those, it is well known that HO-1 exerts anti-inflammatory effects in inflammatory conditions by controlling nuclear factor- $\kappa \mathrm{B}$ activation and production of inflammatory molecules $(35,36)$. Treatment with antioxidants ameliorates the CS-induced neutrophil influx and airway inflammation through the induction of HO-1 (13). Li et al reported that antioxidants downregulate the expression of inflammatory cytokines and upregulate the expression of HO-1 in CSE-stimulated macrophages and bronchial epithelial cells (15). Therefore, HO-1 induction may be useful in the treatment of CS-induced airway inflammation. In the present study, it was confirmed that PP administration upregulated the expression of HO-1 compared with the CS + LPS group. PP more effectively increased HO-1 expression compared with the NC group $(\mathrm{P}<0.01)($ Fig. 5). This result suggests that $\mathrm{PP}$ may be useful in the induction of HO-1 in CS-induced airway inflammation.

Airway epithelial cells are first-defense cells and an important source of inflammatory cytokines and chemokines against CS (12). Similar to the results obtained by PP in vivo, the increased release of inflammatory cytokines and chemokines was markedly decreased with PP administration in CSE-stimulated A549 airway cells (Fig. 6). Nrf2 activation and $\mathrm{HO}-1$ expression were also upregulated with PP treatment in A549 cells (Fig. 7).

Antioxidants have therapeutic as well as preventive potential in the airway inflammatory response of COPD and, therefore, may prove to be a useful therapeutic approach to the treatment of COPD (37). In several studies, antioxidants have been reported to protect against the airway inflammatory response by reducing the influx of inflammatory cells and the levels of inflammatory molecules, and by upregulation of HO-1. Therefore, it was evaluated whether PP, which is a strong antioxidant, exerts a protective effect in airway inflammation. In the present study, PP effectively attenuated airway inflammation by inhibition of neutrophil influx and of inflammatory toxic molecules, such as ROS, which are important pathophysiological characteristics in COPD. These effects of PP were accompanied by Nrf2 activation and HO-1 induction, in vitro as well as in vivo. Therefore, our results suggest that PP may be a valuable therapeutic adjuvant in airway inflammatory diseases, including COPD.

\section{Acknowledgements}

The present study was supported by grants from the KRIBB Research Initiative Program (no. KGM 1221713), the International Biological Material Research Center (no. NRF 2016K1A1A8A01939075) and the Ministry of Health and Welfare (no. HI14C1277) of the Republic of Korea.

\section{References}

1. Vestbo J,Hurd SS, Agustí AG, Jones PW, Vogelmeier C, Anzueto A Barnes PJ, Fabbri LM, Martinez FJ, Nishimura M, et al: Global strategy for the diagnosis, management, and prevention of chronic obstructive pulmonary disease: GOLD executive summary. Am J Respir Crit Care Med 187: 347-365, 2013.

2. Wei J,Fan G,Zhao Hand Li J: Heme oxygenase-1 attenuates inflammation and oxidative damage in a rat model of smoke-induced emphysema. Int J Mol Med 36: 1384-1392, 2015.
3. Tamimi A, Serdarevic D and Hanania NA: The effects of cigarette smoke on airway inflammation in asthma and COPD: Therapeutic implications. Respir Med 106: 319-328, 2012.

4. Lee J, Taneja V and Vassallo R: Cigarette smoking and inflammation: Cellular and molecular mechanisms. J Dent Res 91: 142-149, 2012.

5. Afonso AS, Verhamme KM, Sturkenboom MC and Brusselle GG: COPD in the general population: Prevalence, incidence and survival. Respir Med 105: 1872-1884, 2011.

6. O'Donnell R, Breen D, Wilson S and Djukanovic R: Inflammatory cells in the airways in COPD. Thorax 61: 448-454, 2006.

7. Lee JW, Shin NR, Park JW, Park SY, Kwon OK, Lee HS, Hee Kim J, Lee HJ, Lee J, Zhang ZY, et al: Callicarpa japonica Thunb. attenuates cigarette smoke-induced neutrophil inflammation and mucus secretion. J Ethnopharmacol 175: 1-8, 2015.

8. Lee E, Yun N, Jang YP and Kim J: Lilium lancifolium Thunb. extract attenuates pulmonary inflammation and air space enlargement in a cigarette smoke-exposed mouse model. J Ethnopharmacol 149: 148-156, 2013.

9. Garcia-Rio F, Miravitlles M, Soriano JB, Muñoz L, DuranTauleria E, Sánchez G, Sobradillo V and Ancochea J; EPI-SCAN Steering Committee: Systemic inflammation in chronic obstructive pulmonary disease: A population-based study. Respir Res 11: 63, 2010.

10. Zhou R, Luo F, Lei H, Zhang K, Liu J, He H, Gao J, Chang X, He L, Ji H, et al: Liujunzi Tang, a famous traditional Chinese medicine, ameliorates cigarette smoke-induced mouse model of COPD. J Ethnopharmacol 193: 643-651, 2016.

11. Jung KH, Beak H, Park S, Shin D, Jung J, Park S, Kim J and Bae H: The therapeutic effects of tuberostemonine against cigarette smoke-induced acute lung inflammation in mice. Eur J Pharmacol 774: 80-86, 2016.

12. Victoni T, Gleonnec F, Lanzetti M, Tenor H, Valença S, Porto LC, Lagente V and Boichot E: Roflumilast N-oxide prevents cytokine secretion induced by cigarette smoke combined with LPS through JAK/STAT and ERK1/2 inhibition in airway epithelial cells. PLoS One 9: e85243, 2014.

13. Lee JW, Park HA, Kwon OK, Jang YG, Kim JY, Choi BK, Lee HJ, Lee S, Paik JH,Oh SR, et al: Asiatic acid inhibits pulmonary inflammation induced by cigarette smoke. Int Immunopharmacol 39: 208-217, 2016.

14. Ge LT, Liu YN, Lin XX, Shen HJ, Jia YL, Dong XW, Sun Y and Xie QM: Inhalation of ambroxol inhibits cigarette smoke-induced acute lung injury in a mouse model by inhibiting the Erk pathway. Int Immunopharmacol 33: 90-98, 2016.

15. Li J, Tong D, Liu J, Chen F and Shen Y: Oroxylin A attenuates cigarette smoke-induced lung inflammation by activating Nrf2. Int Immunopharmacol 40: 524-529, 2016.

16. Cheng L, Li F, Ma R and Hu X: Forsythiaside inhibits cigarette smoke-induced lung inflammation by activation of Nrf 2 and inhibition of NF- $\mathrm{B}$. Int Immunopharmacol 28: 494-499, 2015.

17. Osorio-Guarín JA,Enciso-Rodríguez FE, González C,FernándezPozo N, Mueller LA and Barrero LS: Association analysis for disease resistance to Fusarium oxysporum in cape gooseberry (Physalis peruviana L). BMC Genomics 17: 248, 2016.

18. Wu SJ, Tsai JY, Chang SP, Lin DL, Wang SS, Huang SN and Ng LT: Supercritical carbon dioxide extract exhibits enhanced antioxidant and anti-inflammatory activities of Physalis peruviana. J Ethnopharmacol 108: 407-413, 2006.

19. Abdel Moneim AE: Prevention of carbon tetrachloride (CCl4)-induced toxicity in testes of rats treated with Physalis peruviana L. fruit. Toxicol Ind Health 32: 1064-1073, 2016.

20. Al-Olayan EM, El-Khadragy MF, Omer SA, Shata MT, Kassab RB and Abdel Moneim AE: The beneficial effect of cape gooseberry juice on carbon tetrachloride-induced neuronal damage. CNS Neurol Disord Drug Targets 15: 344-350, 2016.

21. Lee JW, Park JW, Shin NR, Park SY, Kwon OK, Park HA, Lim Y, Ryu HW, Yuk HJ, Kim JH, et al: Picrasma quassiodes (D. Don) Benn. attenuates lipopolysaccharide (LPS)-induced acute lung injury. Int J Mol Med 38: 834-844, 2016.

22. Lee JW, Park JW, Kwon OK, et al: NPS2143 inhibits MUC5AC and proinflammatory mediators in cigarette smoke extract (CSE)-stimulated human airway epithelial cells. Inflammation, 2016.

23. Sutherland ER and Martin RJ: Airway inflammation in chronic obstructive pulmonary disease: Comparisons with asthma. J Allergy Clin Immunol 112: 819-827, quiz 828, 2003.

24. Kirkham PA and Barnes PJ: Oxidative stress in COPD. Chest 144: 266-273, 2013. 
25. Rahman I: The role of oxidative stress in the pathogenesis of COPD: Implications for therapy. Treat Respir Med 4: 175-200, 2005.

26. Barnes PJ: Inflammatory mechanisms in patients with chronic obstructive pulmonary disease. J Allergy Clin Immunol 138 : 16-27, 2016.

27. Ng DS, Liao W, Tan WS, Chan TK, Loh XY and Wong WS: Anti-malarial drug artesunate protects against cigarette smokeinduced lung injury in mice. Phytomedicine 21: 1638-1644, 2014.

28. Bodas M, Silverberg D, Walworth K, Brucia K and Vij N: Augmentation of S-nitrosoglutathione controls cigarette smokeinduced inflammatory-oxidative stress and chronic obstructive pulmonary disease-emphysema pathogenesis by restoring cystic fibrosis transmembrane conductance regulator function. Antioxid Redox Signal 27: 433-451, 2017.

29. Gupta I, Ganguly S, Rozanas CR, Stuehr DJ and Panda K: Ascorbate attenuates pulmonary emphysema by inhibiting tobacco smoke and Rtp801-triggered lung protein modification and proteolysis. Proc Natl Acad Sci USA 113: E4208-E4217, 2016.

30. Chen B, You WJ, Xue S, Qin H, Zhao XJ, Zhang M, Liu XQ, Zhu SY and Jiang HD: Overexpression of farnesoid X receptor in small airways contributes to epithelial to mesenchymal transition and COX-2 expression in chronic obstructive pulmonary disease. J Thorac Dis 8: 3063-3074, 2016.

31. Li D, Hu J, Wang T, Zhang X, Liu L, Wang H, Wu Y, Xu D and Wen F: Silymarin attenuates cigarette smoke extract-induced inflammation via simultaneous inhibition of autophagy and ERK/p38 MAPK pathway in human bronchial epithelial cells. Sci Rep 6: 37751, 2016
32. Li D, Xu D, Wang T, Shen Y, Guo S, Zhang X, Guo L, Li X, Liu L and Wen F: Silymarin attenuates airway inflammation induced by cigarette smoke in mice. Inflammation 38: 871-878, 2015.

33. Wu X, Song M, Rakariyatham K, Zheng J, Guo S, Tang Z, Zhou S and Xiao H: Anti-inflammatory effects of 4'-demethylnobiletin, a major metabolite of nobiletin. J Funct Foods 19: 278-287, 2015.

34. Yao W, Luo G, Zhu G, Chi X, Zhang A, Xia Z and Hei Z: Propofol activation of the Nrf2 pathway is associated with amelioration of acute lung injury in a rat liver transplantation model. Oxid Med Cell Longev 2014: 258567, 2014.

35. Lee JW, Bae CJ, Choi YJ, Kim SI, Kwon YS, Lee HJ, Kim SS and Chun W: 3,4,5-Trihydroxycinnamic acid inhibits lipopolysaccharide (LPS)-induced inflammation by Nrf2 activation in vitro and improves survival of mice in LPS-induced endotoxemia model in vivo. Mol Cell Biochem 390: 143-153, 2014.

36. Li RJ, Gao CY, Guo C, Zhou MM, Luo J and Kong LY: The Antiinflammatory activities of two major withanolides from Physalis minima via acting on NF- $\mathrm{KB}, \mathrm{STAT} 3$, and HO-1 in LPS-stimulated RAW264.7 cells. Inflammation 40: 401-413, 2017.

37. Domej W, Oettl K and Renner W: Oxidative stress and free radicals in COPD - implications and relevance for treatment. Int J Chron Obstruct Pulmon Dis 9: 1207-1224, 2014. 